\title{
ALGEBRAIC CRITERION ON QUASICONFORMAL EQUIVALENCE OF RIEMANN SURFACES
}

\author{
MITSURU NAKAI
}

\section{Introduction}

1. Various strides have been done to characterize the conformal structure of Riemann surfaces by the algebraic structure of some appropriate function algebras on them (cf. Bers [2], Rudin [29], Royden [26], [28], Heins [7], Kakutani [12], Wermer [33] etc.). In this paper we discuss, corresponding to the above, the problem to determine the quasiconformal structure of Riemann surfaces by the algebraic structure of some function algebras on them.

2. Quasiconformal equivalence. Following Pfluger-Ahlfors-Mori ([21], [1], [14]), we say that a mapping $T$ of a Riemann surface $R$ onto another Riemann surface $R^{\prime}$ is a quasiconformal mapping of $R$ onto $R^{\prime}$ if it is a topological mapping of $R$ onto $R^{\prime}$ and if its maximal dilatation $K(T)$ is finite. Here $K(T)$ is defined by the following: $K(T)=\inf \left\{\lambda ; \lambda^{-1} \bmod Q \leqq \bmod T Q \leqq \lambda \bmod Q\right.$ for all $Q$ in $\mathbf{Q}(R)\}$, where $\mathbf{Q}(R)$ denotes the totality of quadrilaterals on $R$ and $\bmod$ $Q$ denotes the modulus of $Q$.

Now consider two Riemann surfaces $R$ and $R^{\prime}$. If there exists a quasiconformal mapping of $R$ onto $R^{\prime}$, then $R$ and $R^{\prime}$ are said to be quasiconformally equivalent or to have the same quasiconformal structure.

3. Royden's algebra. Let $R$ be a Riemann surface and $M(R)$ be the totality of complex-valued functions $f$ on $R$ satisfying the following three conditions :

(M. 1) $f$ is absolutely continuous in the sense of Tonelli ${ }^{1}$;

Received October 29, 1959.

1) A function $F(x, y)$ on a rectangle $[a, b ; c, d]$ is called absolutely continuous in the sense of Tonelli (abbreviated as a.c.T.) if $F(x, y)$ is absolutely continuous in $x$ varying in $[a, b]$ for almost all fixed values $y$ in $[c, d]$ and if the corresponding fact holds by interchanging $x$ and $y$ and, further, if $F_{x}$ and $F_{y}$ are locally integrable. (For details, refer to $[22,30,34]$.) This notion is carried over Riemann surfaces by using local parameters. 
(M. 2) $f$ is bounded on $R$ in the absolute value;

(M. 3) Dirichlet integral of $f$ over $R$ is finite.

We define the algebraic operations in $M(R)$ as usual, that is, for $f$ and $g$ in $M(R)$ and for a complex number $a,(f+g)(p)=f(p)+g(p),(f g)(p)=f(p) g(p)$ and $(a f)(p)=a(f(p))$ at every point $p$ in $R$. Then $M(R)$ becomes an algebra over the complex number field. This function algebra $M(R)$ is called Royden's algebra associated with $R^{2)}$ Some applications of Royden's algebra to the function theory were given by Royden [24], [25], [26], S. Mori [15], [16], Mori-Ôta [17] and the present author [18], [19].

4. Main theorems. The main result of this paper is that the algebraic structure of the Royden's algebra $M(R)$ associated with a Riemann surface $R$ determines the quasiconformal structure of $R{ }^{3)}$ namely:

TheOREM. Two Riemann surfaces $R$ and $R^{\prime}$ are quasiconformally equivalent if and only if their Royden's algebras $M(R)$ and $M\left(R^{\prime}\right)$ are algebraically isomorphic.

The Royden's algebra $M(R)$ can be normed by

$$
\|f\|=\sup _{R}|f|+\sqrt{\iint_{R}|\operatorname{grad} f|^{2} d x} d y .
$$

Using this norm, we can state ${ }^{4)}$

THEOREM. Two Riemann surfaces $R$ and $R^{\prime}$ are conformally ${ }^{5)}$ equivalent if and only if $M(R)$ and $M\left(R^{\prime}\right)$ are normed algebraically isomorphic. $\left.{ }^{6}\right)$

5. ${ }^{7)}$ In Chapter I, we give a general theorem on some subalgebra of bounded continuous function algebra. Our main tool is the notion of unremovability of

2) The original definition of Royden's algebra due to Royden [24] is as follows: let $B D(R)$ be the totality of the complex-valued bounded piecewise smooth functions defined on $R$ with finite Dirichlet integrals. Then $B D(R)$ can be normed by $\|f\|=\sup _{R}|f|$ $+\sqrt{\iint_{R}|\operatorname{grad} f|^{2} d x d y}$. Then the completed algebra of $B D(R)$ by this norm is called Royden's algebra. As for the coincidence of both definitions, refer to [18].

3) An improvement of the author's previous result, Theorem 4 in [18].

4) An extension of the author's previous result in [19], in which this was proved under the condition that $R$ and $R^{\prime}$ are compact.

5) Including both of direct and indirect ones.

6) i.e., this means that $M(R)$ and $M\left(R^{\prime}\right)$ are algebraically isomorphic and this isomorphism preserves the norm $\|f\|$.

7) The result stated in this paper was published in [20] without proofs. 
a point for a family of functions with some property. In Chapter II, a quantity is defined for some topological mapping and we characterize the quasiconformality of this topological mapping by using this quantity. In Chapter III, the results above mentioned are proved. By using the algebra $M^{n}(R)$ consisting of all functions in $M(R)$ which are $n$-fold continuously differentiable $(\mathrm{n}=1,2$, ...,, , similar results as above are proved for $M^{n}(R)$ instead of $M(R)$.

The author wishes to express here his hearty thanks to Prof. K. Noshiro for his kind encouragements and guidances, and also to Prof. T. Kuroda for his valuable discussions.

\section{Some subalgebras of continuous function algebras}

1. In this chapter we treat the problem characterizing a given topological space $S$ by an algebra of some continuous functions defined on $S$. It is well known that the total algebra $C(S)$ of all continuous functions defined on $S$ determines $S$ if $S$ is a compact Hausdorff space (Čech [5]). As an extension, Hewitt [8] and Shirota [32] proved that $C(S)$ determines $S$ if and only if $S$ is a $Q$-space. For arbitrary spaces $S$ and for arbitrary subalgebras $P(S)$ of $C(S)$. it is not always true that $P(S)$ determines $S$. But under some conditions on $S$ and on the structure of $P(S)$, it is known that the same holds (cf. Pursell [23], Isiwata [10]).

In this chapter we shall only consider the locally compact Hausdorff spaces S. For locally compact spaces S, Shanks [31] and Ishii [9] proved that the subalgebra $C_{0}(S)$ of $C(S)$ consisting of all functions with compact carriers determines $S$. Some generalization of this was given by Isiwata [11].

We shall consider some appropriate property $P$ on functions of $C(S)$ and we shall prove that the algebra $P(S)$ consisting of functions in $C(S)$ with a property $P$ determines $S$ if $S$ has no removable point with respect to the property $P$. These discussions play important roles to study Royden's algebra.

2. A property on continuous functions. We denote by $\mathbf{S}=\{S\}$ the totality of locally compact Hausdorff spaces $S$. Let $f$ be a complex-valued continuous function defined on $S$. We consider the totality $C$ of such a pair $(f, S)$, i.e., $C=\{(f, S) ; S$ is in $\mathbf{S}$ and $f$ is a continuous function defined on $S\}$. Let $P$ be a subfamily of $C$. We say that a continuous function $f$ defined on $S$ has a property $P$ if $(f, S)$ belongs to $P$ and that $P$ is a property on continuous func- 
tions. By $P(S)$ we denote the set $\{f ;(f, S)$ belongs to $P\}$. Now we consider the following conditions (P.1)-(P.7) on a property $P$ :

(P.1) $P(S)$ is an algebra over the complex number field with the constant function 1 ;

(P.2) $P(S)$ separates points in $S$ strongly, i.e., for any neighborhood $V$ of any point $p$ in $S$, there exists a function $f$ in $P(S)$ such that $f=0$ on $S-V$ and 1 on a neighborhood of $p$ contained in $V$;

(P. 3) $P(S)$ is inverse-closed, i.e., if $f$ is in $P(S)$ and inf $|f|>0$, then the function $1 / f$ belongs to $P(S)$;

(P.4) $P(S)$ is self-adjoint, i.e., if $f$ belongs to $P(S)$, then $f^{*}$ belongs to $P(S)$, where $f^{*}(p)=\overline{f(p)}$ (the complex conjugate of $f(p)$ ) for all points $p$ in $S$;

(P.5) every function $f$ in $P(S)$ is bounded in the absolute value;

(P.6) $P$ is monotone, i.e., if $S^{\prime}$ belongs to $S$ and $S$ is an open subset of $S^{\prime}$, if $(f, S)$ belongs to $P$ and if $f$ vanishes on a neighborhood of the relative boundary of $S$ with respect to $S^{\prime}$, then $\left(f^{\prime}, S^{\prime}\right)$ also belongs to $P$, where $f^{\prime}=f$ on $S$ and $f^{\prime}=0$ on $S^{\prime}-S$. Conversely, $(f, S)$ belongs to $P$ if $\left(f, S^{\prime}\right)$ belongs to $P$.

We say that a topological mapping $T$ of $S$ onto $S^{\prime}$ has property $P$ when ( $f \circ T^{-1}, S^{\prime}$ ) belongs to $P$ if and only if $(f, S)$ belongs to $P$. A mapping $\sigma$ of $P(S)$ onto $P\left(S^{\prime}\right)$ is said to be induced by a topological mapping $T$ locally if $T$ is a topological mapping of an open subset $S_{1}$ of $S$ onto an open subset $S_{1}^{\prime}$ of $S^{\prime}$ and $f^{\circ}(T p)=f(p)$ holds for any $p$ in $S_{1}$ and for any $f$ in $P(S)$, where $f^{\circ}$ is the image of $f$ by the mapping $\sigma$. By using these terminologies, our additional condition is stated as follows:

(P.7) if (P.1) is satisfied and if an algebraic isomorphism of $P(S)$ onto $P\left(S^{\prime}\right)$ is induced by a topological mapping $T$ locally, then $T$ has the property $P$.

We shall say that a property $P$ satisfying the conditions (P.1), (P.2), (P.3), (P.4), (P.5), (P.6) and (P.7) is an admissible property on continuous functions.

Examples of admissible properties are $B, B_{0}$, and $B_{\infty}$, where $B=\{(f, S)$; $(f, S)$ is in $C$ and $f$ is bounded in the absolute value on $S\}, B_{0}=\{(f, S) ;(f, S)$ is in $C$ and $f$ is constant outside a compact subset of $S\}$ and $B_{\infty}=\{(f, S) ;(f, S)$ is in $C$ and there exists a constant $c_{f}$ such that for any $\varepsilon>0$ there exists a 
compact set outside which we have $\left.\left|f-c_{f}\right|<\varepsilon\right\}$.

3. Premovability. Let $S$ be in $S$ and $P$ be a property on continuous func. tions. A point $p$ in $S$ is said to be Premovable if the following condition is satisfied: for any pair $(f, S-\{p\})$ belonging to $P$, there exists a pair $\left(f^{\prime}, S\right)$ belonging to $P$ such that $f(q)=f^{\prime}(q)$ holds for any $q$ in $S-\{p\}$. In other words, choosing a suitable value of $f$ at $p$, we can extend $f$ to the whole space $S$ such that $(f, S)$ is contained in $P$. We consider the subfamily $\mathbb{S}_{P}$ of $\mathbf{S}$ defined by the following: $S$ is in $S_{P}$ if and only if $S$ has no $P$-removable point.

We state an example. Let $S$ be in $S$ and let $S$ be non-compact. Let $\beta S$ be the Čech compactification of $S$, i.e., the compact Hausdorff space containing $S$ as its open dense subset and every function in $B(S)$ is extended over $\beta S$ so as to be continuous on $\beta S$. Then each point of $\beta S-S$ is $B$-removable and $\beta S$ does not belong to $\mathbf{S}_{B}$. On the other hand, any manifold belongs to $\mathbf{S}_{B}$ or more generally, each space in $\mathbf{S}$ whose each point is $C_{\delta}$ belongs to $\mathbf{S}_{B}$.

4. Theorem 1.1. Let $P$ be an admissible property on continuous functions and, $S$ and $S^{\prime}$ belong to $S_{P}$. Assume that $\sigma$ is an algebraic isomorphism of $P(S)$ onto $P\left(S^{\prime}\right)$. Then there exists a topological mapping $T$ of $S$ onto $S^{\prime}$ such that $f^{\circ}(p)=f\left(T^{-1}(p)\right)$ for any point $p$ in $S^{\prime}$ and for any function $f$ in $P(S)$, where $f^{\circ}$ denotes the image of $f$ under the mapping $\sigma$.

5. Pucompactification. For the proof of Theorem $\mathrm{I}$.1, we need same preliminaries. I.et $P$ be a property satisfying (P. 1), (P.2), (P.3), (P.4) and (P.5). For each space $\mathrm{S}$ in $\mathrm{S}$ we associate a space $\hat{\mathrm{S}}=\hat{\mathrm{S}}_{P}$, which will be called $P$. compactification of $S$, satisfying the following three conditions:

(S.1) $\hat{S}$ is a compact Hausdorff space containing $S$ as its topological sub. space ;

(S. 2) $\hat{S}$ is open and dense in $S$;

(S.3) for each $f$ belonging to $P(S)$ there exists a continuous function $\hat{f}$ defined on $\hat{S}$ such that $\hat{f}(p)=f(p)$ for any point $p$ in $S$, i.e., $f$ can be extended continuously to $\hat{S}$.

We say that a complex-valued functional $X$ on $P(S)$ is a $P$-character on $S$ or simply a character on $P(S)$ if it satisfies the following conditions: for functions $f$ and $g$ in $P(S)$ and for any complex numbers $a$ and $b$, 
(X. 1) $X(1)=1$;

(X.2) $X(a f+b g)=a X(f)+b X(g), X(f g)=X(f) X(g)$;

(X.3) $|X(f)| \leqq n(f)$, where $n(f)=\sup _{s}|f|$.

A point $p$ in $S$ determines a character $X_{p}$ on $P(S)$ defined by $X_{p}(f)=f(p)$ on $P(\mathrm{~S})$. By (P.2), $p \neq q$ implies $X_{p} \neq X_{q}$. We say that a character $X_{p}$ is determined by $p$. We denote by $S^{*}=S_{P}^{*}$ the totality of $P$-characters on $S$ and we call $S_{P}^{*}$ the $P$-character space of $S$. First we prove the following fundamental

Lemma I.1. For any locally compact space $S$, there exists a unique $P$ compactification $\hat{S}$ of $S$.

Proof. Let $\hat{S}$ be the set of points $x, y, \ldots$ containing $S$ as its subset such that there exists a one-to-one mapping $i$ of the $P$-character space $S^{*}$ of $S$ onto $\hat{S}$. Moreover, we assume that $i\left(X_{p}\right)=p$, where $X_{p}$ is the character determined by $p$. We denote by $\hat{P}(\hat{S})$ the totality of functions $\hat{f}$ on $\hat{S}$ defined by $\hat{f}(x)$ $=\left(i^{-1}(x)\right)(f)$. Now we consider $P(S)$ as a normed space with the norm $n(f)$. Then by (X.3), $S^{*}$ is contained in the dual space $P(S)^{*}$ of $P(S)$ and further $S^{*}$ is contained in the unit sphere of $P(S)^{*}$. It is easy to see that $S^{*}$ is closed in $P(S)^{*}$ with respect to the weak topology $\sigma\left(P(S)^{*}, P(S)\right)$ of $P(S)^{*}$ as func tionals. Then the well known Tychonoff-Kakutani theorem yields the compactness of $S^{*}$. Hence $S^{*}$ is a compact Hausdorff space. We introduce a topology into $\hat{S}$ by $i$ such that $i$ is a homeomorphism between $S^{*}$ and $\hat{S}$. It is easily seen that the relative topology of $S$ with respect to $\hat{S}$ is coincident with the proper topology of $S$ as a locally compact Hausdorff space. Hence we have shown that (S.1) is satisfied by $\hat{S}$. By this topology, it is clear that $\hat{P}(\hat{S})$ is the family of functions $\hat{f}$ which are continuous on $\hat{S}$. Moreover, $\hat{f}(p)=f(p)$ on $S$ and $\hat{n}(\hat{f}) \leqq n(f)$, where $\hat{n}(f)=\sup _{\hat{S}}|\hat{f}(x)|$. Thus $\hat{f}$ is a bounded continuous extension of $f$ on $S$ to $\hat{S}$. Thus (S.3) is also satisfied by $\hat{S}$.

Now it is clear that $\hat{P}(\hat{S})$ separates points in $\hat{S}$. For, if $x$ and $y$ are in $\hat{S}$ and $x \neq y$, then $X=i^{-1}(x)$ and $Y=i^{-1}(y)$ are distinct. Hence there exists a function $f$ in $P(S)$ such that $X(f) \neq Y(f)$, which shows $\hat{f}(x) \neq \hat{f}(y)$. Thus $\hat{P}(\hat{S})$ is an algebra with 1 which separates points in $S$. Thus by the WeierstrassStone approximation theorem (Bourbaki $[4]), \hat{P}(\hat{S})$ is dense in $C(\hat{S})$ with the topology induced by the norm $\hat{n}(f)$.

If $S$ is not dense in $\hat{S}$, there exists a function $g$ in $C(\hat{S})$ such that $g \neq \dot{0}$ 
and $g=0$ on $S$. By the above we can find a sequence $\left\{\hat{f}_{m}\right\}$ in $\hat{p}(\hat{S})$ such that $\hat{f}_{m}$ converges uniformly to $g$. Thus we may assume $\left|f_{m}(p)\right|<1 / m$ on $S$. As already stated, $\hat{f}_{m}=f_{m}$ on $S$ and $\hat{n}(\hat{f}) \leqq n\left(f_{m}\right) \leqq 1 / m$. Thus $\left\{\hat{f}_{m}\right\}$ converges to 0 uniformly. So $g \equiv 0$, which is a contradiction. Hence $S$ is dense in $\hat{S}$. Thus, to show that $S$ satisfies (S.2), we have only to prove that $S$ is open in $\hat{S}$ or that $\hat{S}-S$ is closed.

To verify this, we first prove that $x$ belongs to $\hat{S}-S$ if and only if $\hat{f}(x)$ $=0$ for all $f$ in $P(S)$ with compact carrier. The "if part" of the above statement is evident. Conversely, we shall prove that $\hat{f}(x)=0$ for all $f$ in $P(S)$ with compact carrier if $x$ belongs to $\hat{S}-S$. Contrary to the assertion, suppose that there exists a function $f_{0}$ in $P(S)$ with compact carrier such that $\hat{f}_{0}(x) \neq 0$. We may assume $f_{0} \geqq 0$. Put $R=\left\{p ; p\right.$ is in $S$ and $\left.f_{0}(p)>0\right\}$ and $Z=\{f ; f$ is in $P(S)$ with compact carrier contained in the closure of $R$ in $S$, and $\hat{f}(x)=0\}$. For each point $p$ in $R$, we can find $f_{p}$ in $Z$ such that $f_{p}(p) \neq 0$. If this is not the case, $(g-g(x)) f_{0}$ is in $Z$ for any $g$ in $P(S)$ and so $(g(p)-\hat{g}(x)) f_{0}(p)=0$. Thus $g(p)=\hat{g}(x)$ holds for all $g$ in $P(S)$, which shows that $X_{p}(g)=i^{-1}(x)(g)$ or $x=p$, which is a contradiction. Thus we can find $f_{p}$ in $Z$ such that $f_{p}(p) \neq 0$. Clearly, we may assume $f_{p} \geqq 0$ and $f_{p}(p)=1$. Let $h_{0}=\left(f_{0}-\hat{f}_{0}(x)\right)^{2} /\left(\hat{f}_{0}(x)\right)^{2}$. Then $h_{0}(x)=0$ and $h_{0} \geqq 0$. Moreover, $h_{0}(p) \geqq 1 / 2$ on $S-R^{\prime}$, where $R^{\prime}$ is a compact set in S. We can find a finite number of points in $R^{\prime}$, say $p_{j}$, and $f_{p_{j}}$ in $Z$ such that $\sum_{j} f_{p_{j}} \geqq 1 / 2$ on $R^{\prime}$. Then $h=h_{0}+\sum_{j} f_{p_{j}} \geqq 1 / 2$ and $\hat{h}(x) \geqq 1 / 2$ and on the other hand $\hat{h}(x)=\hat{h}_{0}(x)+\sum_{j} \hat{f} p_{j}(x)=0$. This is a contradiction. Therefore, $\hat{S}-S$ coincides with the intersection of zero-sets of continuous functions. So $S$ is open in $\hat{S}$. Thus the proof of the existence of the $P$-compactification of $S$ is established.

Finally we show the unicity of $P$-compactification. Let $\hat{S}_{j}(j=1,2)$ be $P$. compactifications of $S$. By (S.2), the extensions $\hat{f}_{j}$ of $f$ in $P(S)$ to $\hat{S}_{j}$ is unique. Thus the correspondence $i_{j}: f \rightarrow \hat{f}_{j}$ gives an algebraic isomorphism of $P(S)$ onto $\left\{\hat{f}_{j}\right\}$. Thus $i_{2} \circ i_{1}^{-1}: \hat{f}_{1} \rightarrow \hat{f}_{2}$ gives an algebraic isomorphism of $\left\{\hat{f}_{1}\right\}$ onto $\left\{\hat{f}_{2}\right\}$. It is easy to see that $\left\{\hat{f}_{j}\right\}$ are dense in $C\left(\hat{S}_{j}\right)$ with respect to the uniform topology. It is clear that $i_{1}$ and $i_{2}$, and hence $i_{2} \circ i_{1}^{-1}$ are isometric with respect to the uniform norm. So the isomorphism of $\left\{\hat{f}_{1}\right\}$ onto $\left\{\hat{f}_{2}\right\}$ induces an isomorphism of $C\left(\hat{S}_{1}\right)$ onto $C\left(\hat{S}_{2}\right)$. As $\hat{S}_{j}$ are compact, the classical Čech theorem implies that $\hat{S}_{1}$ and $\hat{S}_{2}$ are homeomorphic. 
This completes the proof of Lemma 1.1.

We state some examples of $P$-compactifications. As is already stated, $B$ compactification $\hat{S}_{B}$ of a locally compact space $S$ is the Čech-compactification $\beta S$. Both $B_{0^{\circ}}$ compactification and $B_{\infty}$-compactification of a locally compact space $S$ are the Alexandroff-compactification $S_{\infty}$ which is formed by adding one point $\infty$ to $S$. Now we introduce a new property. Let $B_{1}$ be the subfamily of $C$ such that $(f, S)$ belongs to $B_{1}$ if and only if $(f, S)$ belongs to $B$ and, for each positive number $\varepsilon$, there exists a compact subset $K$ and a family of constants $\left\{c_{D}\right\}$, where $\{D\}$ is the totality of components of $S-K$, such that $\left|f(p)-c_{D}\right|$ is smaller than $\varepsilon$ on $D$ for each $D$ in $\{D\}$. Then $B_{1}$ is the property satisfying (P.1), (P.2), (P.3), (P.4), and (P.5). The $B_{1}$-compactification of a locally compact space $S$ is nothing but the Kerékjártó-Stoilow compactification of $S$ (cr. Royden [27]).

As is easily seen from the proof of Lemma 1.1, we get the following representation theorem of $P$-characters:

Lemma I.2. For any P-character $X$ on $S$, there exists a point $x$ in the $P$ compactification $\hat{S}$ of $S$ such that $X(f)=\hat{f}(x)$ for all $f$ in $P(S)$.

Next we state a simple and useful lemma:

Lemma 1.3. Any algebraic isomorphism of of $P(\mathrm{~S})$ onto $P\left(\mathrm{~S}^{\prime}\right)$ is isometric with respect to the uniform norm, that is, $n\left(f^{\sigma}\right)=n(f)$ for all $f$ in $P(S)$.

Proof. Let $f$ belong to $P(S)$ and $s(f)$ be the spectra of $f$, where a complex number $a$ belongs to $s(f)$ if and only if $f-a$ is not inversible in $P(S)$ and hence $\inf _{s}|f-a|=0$ by (P.3). Then it is easy to see that $n(f)=\sup \{|a|$; $a$ belongs to $s(f)\}$ and that $s\left(f^{\circ}\right)=s(f)$. From these, our assertion follows.

6. Proof of Theorem I. 1. We consider P-compactifications $\hat{S}$ and $\hat{S}^{\prime}$ of $S$ and $S^{\prime}$, respectively. We construct a topological mapping $T$ of $\hat{S}$ onto $\hat{S}^{\prime}$ as follows. Take a point $x$ in $\hat{S}$. Then we can find a $P$-character $X$ in the $P$ character space $S^{*}$ of $S$ such that $X(f)=\hat{f}(x)$, where $\hat{f}$ is the topological continuation, to $\hat{S}$, of $f$ belonging to $P(S)$. To this $X$, we define a functional $Y$ on $P\left(S^{\prime}\right)$ by $Y(f)=X\left(f^{o-1}\right)$, where $f$ is in $P\left(S^{\prime}\right)$. Then by Lemma I.3, it is easily verified that $Y$ belongs to the $P$-character space $S^{* *}$ of $S^{\prime}$. Using Lemma I.2, we can find a point $y$ in $\hat{S}^{\prime}$ such that $Y(f)=\hat{f}(y)$, where $\hat{f}$ is the topo- 
logical continuation, to $\hat{S}^{\prime}$, of $f$ belonging to $P\left(\hat{S}^{\prime}\right)$. These give rise to a mapping $T: x \rightarrow y$ of $\hat{S}$ into $\hat{S}^{\prime}$. This is clearly one-to-one and onto. Moreover, by the definition of topologies in $\hat{S}$ and $\hat{S}^{\prime}$, we see immediately that $T$ is bicontinuous. To any function $f$ in $P(S)$ and for any point $p$ in $S$, we have the identity

$$
\hat{f}^{\sigma}(T p)=f^{(}(p) \text {. }
$$

Next we prove that the image $T S$ of $S$ under the mapping $T$ is $S^{\prime}$. Contrary to the assertion, suppose that there exists a point $p$ in $S$ which is sent to a point $x^{\prime}$ in $\hat{S}^{\prime}-S^{\prime}$, i.e., $T p=x^{\prime}$. Let $U$ be an open neighborhood of $p$ contained in $S$ with its closure and let $U^{\prime}$ be the image $T U$ of $U$ under $T$. We set $V^{\prime}=U^{\prime} \cap S^{\prime}$ which is a non-void open set in $S^{\prime}$, as $S^{\prime}$ is a dense open subset of $\hat{S}^{\prime}$, and let $V$ be the counter image $T^{-1} V^{\prime}$ of $V^{\prime}$ under $T$. Clearly $V$ is contained in $U$. By the hypothesis that $S$ is contained $\mathbf{S}_{P}$, there exists a function $F$ on $S-\{p\}$ such that $(F, s-\{p\})$ is contained in $P$ but no pair $(g, S)$ exists such that $(g, S)$ is contained in $P$ and $g(q)=F(q)$ on $S-\{p\}$. By the condition (P.2), there exists a function $d(q)$ on $S$ such that $(d, S)$ belongs to $P$ and $d(q)=1$ on a neighborhood of $p$ contained in $U_{1}$, where $U_{1}$ is a neighborhood of $p$ contained in $U$ with its closure, and $d(q)=0$ outside $U_{1}$. We define the function $G$ on $S-\{p\}$ by $G(q)=d(q) F(q)$ on $S-\{p\}$. Then $(G, S-\{p\})$ belongs to $P$. The function $G$ cannot be continued to $p$ such that $(G, S)$ belongs to $P$. In fact, suppose that $G$ can be extended to $S$ and $(G, S)$ belongs to $P$. Since $(1-d) F$ vanishes on a neighborhood of $p$ and $((1-d) F, S-\{p\})$ belongs to $P, \quad((1-d) F, S)$ belongs to $P$ by (P.6). As $(F, S-\{p\})$ $=(G+(1-d) F, S-\{p\})$, we see that $(F, S)$ belongs to $P$ by $(P .1)$, which is a contradiction. Thus $G$ cannot be extended to $S$. Hence we have found a function $G$ defined on $S-\{p\}$ satisfying

(i ) $(G, S-\{p\})$ belongs to $P$;

(ii) $G$ cannot be continued to $p$ such that $(G, S)$ belongs to $P$;

(iii) $G$ vanishes outside a neighborhood $U_{1}$ of $p$, where $U_{1}$ is contained in $U$ with its closure.

Here we notice that $G$ vanishes on $\hat{S}-S$. Now we consider a function $G^{\prime}$ defined on $\hat{S}^{\prime}-\left\{x^{\prime}\right\}$ and a fortiori on $S^{\prime}$ by $G^{\prime}(y)=G\left(T^{-1}(y)\right)$ for $y$ in $\hat{S}^{\prime}-\left\{x^{\prime}\right\}$. As $T$ is a topological mapping of the open subset $V$ of $S$ onto the open subset $V^{\prime}$ of $S^{\prime}$ and as $f^{\prime}(T p)=f(p)$ on $V^{\prime}$, i.e., $T$ induces $\sigma$ locally, so $T$ has the 
property $P$ by (P.7). Thus we can conclude that $\left(G\left(T^{-1}(y)\right), V^{\prime}\right)$ belongs to $P$. Let $q^{\prime}$ be a point in the boundary of $V^{\prime}$ relative to $S^{\prime}$. Then $g=T^{-1} q^{\prime}$ is a boundary point of $V$ relative to $\hat{S}$. Since $U$ is relatively compact in $S$ and $V$ is contained in $U$, the point $q$ belongs to $S$. In this case $q$ cannot be in $U$. Contrary to the assertion, if $q$ is in $U$, then $T U$ contains $q^{\prime}$ and hence $T U \cap S^{\prime}$ $=V^{\prime}$ contains $q^{\prime}$, which contradicts the assumption that $q^{\prime}$ is in the boundary of the open set $V^{\prime}$. Thus $q$ is outside $U$. Hence we can choose a neighborhood $W^{\prime}$ of $q^{\prime}$ such that $T^{-1} W^{\prime}$ is outside $U_{1}$, since the closure of $U_{1}$ is compact in $U$.

Thus we can find a neighborhood $W^{\prime}$ of $q^{\prime}$ such that $T^{-1} y$ belongs to $\hat{S}-U_{1}$ if $y$ belongs to $\hat{S}^{\prime}-W^{\prime}$. If $y$ belongs to $V^{\prime} \cap W^{\prime}$, we see by (iii) that $G\left(T^{-1}(y)\right)=0$. Thus $G\left(T^{-1}(y)\right)$ vanishes on a neighborhood of the relative boundary of $V^{\prime}$ with respect to $S^{\prime}$. Hence, by (P.6), $\left(G^{\prime}, S^{\prime}\right)$ belongs to $P$. Thus we can find a function $g$ in $P(S)$ such that the image of $g$ by $\sigma$ is $G^{\prime}$. By the definition of $T$ we have the identity $G^{\prime}(T q)=g(q)$ for every point $q$ in $S-\{p\}$. By the definition of $G^{\prime}$, it holds the identity $G^{\prime}(T q)=G(g)$ for every point $q$ in $S-\{p\}$. Thus $g$ coincides with $G$ on $S-\{p\}$, which means that $G$ can be continued to $p$ such that it belongs to $P(S)$. This contradicts the assumption (ii) on $G$. This contradiction arises from our assumption $T p=x^{\prime}$ for some points $p$ in $S$ and $x^{\prime}$ in $S^{\prime}-S^{\prime}$. Thus we have proved the inclusion $T S \subset S^{\prime}$. By the similar manner we can show the converse inclusion $T^{-1} S^{\prime} \subset S$. Thus we get the required identity $T S=S^{\prime}$ and so $T$ is a homeomorphism of $S$ onto $S^{\prime}$ and the relation $f^{\sigma}(T p)=f(p)$ holds for any function $f$ in $P(S)$ and for any point $p$ in $S$.

This completes the proof of Theorem I. 1 .

Remark. For later use, we notice the following fact. Let $\sigma$ be an algebraic isomorphism of $P(S)$ onto $P\left(S^{\prime}\right)$, where $P$ is a property on continuous functions satisfying (P.1), (P.2), (P.3), (P.4) and (P.5). Let $T$ induce $\sigma$ locally in $U$ and $U^{\prime}$, that is, $T$ is a topological mapping of an open subset $U$ of $S$ onto an open subset $U^{\prime}$ of $S^{\prime}$ and $f^{\prime}(T p)=f(p)$ on $U$ for all $f$ in $P(S)$. If the sequences $\left\{p_{n}\right\}$ and $\left\{p_{n}^{\prime}\right\}$ in $U$ and $U^{\prime}$, satisfying $T p_{n}=p_{n}^{\prime}$, converges to points $p$ and $p^{\prime}$ in the relative boundary of $U$ and $U^{\prime}$ with respect to $S$ and $S^{\prime}$ respectively, then we can find neighborhoods $W$ and $W^{\prime}$ of $p$ and $p^{\prime}$ in $S$ and $S^{\prime}$ respectively and $T$ can be extended to a topological mapping of $W \cup U$ onto 
$W^{\prime} \cup U^{\prime}$ such that $f^{\circ}(T p)=f(p)$ for all $p$ in $W \cup U$, i.e., the extended $T$ induces. $\sigma$ locally on $W \cup U$ and $W^{\prime} \cup U^{\prime}$.

The proof of this fact is essentially contained in the first part of the proof of Theorem I. 1.

\section{Norm of a certain homeomorphism}

1. Definition of qussiconformal mappings. Let $T$ be a topological mapping of a Riemann surface $R$ onto another Riemann surface $R^{\prime}$. Consider the quantity $K(T)$ defined for $T$ as follows :

$$
K(T)=\inf \left\{\lambda ; \lambda^{-1} \bmod Q \leqq \bmod T Q \leqq \lambda \bmod Q, \text { for all } Q \text { in } \mathbf{Q}\right\},
$$

where $\mathbf{Q}$ denotes the totality of quadrilaterals $\{Q\}$ contained in $R$ and $\bmod \boldsymbol{Q}$ denotes the module of $Q$. Clearly the inequality $1 \leqq K(T) \leqq \infty$ holds. This quantity $K(T)$ is called the maximal dilatation of $T$. If $K(T)$ is finite, then we say that $T$ is a quasiconformal mapping. This definition is due to Ahlfors [1] (see also Pfluger [21] and Mori [14]).8)

2. We state another definition of the quasiconformal mapping which is convenient for our purposes. Corresponding to $K(T)$, we consider the quantity

$$
K^{*}(T)=\inf \left\{\lambda ; \lambda^{-1} \bmod A \leqq \bmod T A \leqq \lambda \bmod A, \text { for all } A \text { in } \mathbf{A}\right\},
$$

where $\mathbf{A}$ denotes the totality of annuli $A$ which are Jordan domains contained in some simply connected domains on $R$ and $\bmod A$ denotes the module of $A$. Clearly we have the relation $1 \leqq K^{*}(T) \leqq \infty$. Then we get the following criterion of the quasiconformality [18]:

Theorem II.1.9) A topological mapping $T$ of a Riemann surface $R$ onto another Riemann surface $R^{\prime}$ is a quasiconformal mapping if and only if $K^{*}(T)$ is finite.

In the particular case that $K^{*}(T)$ is 1 , we have the following (see Nakai [19]) :

Theorem II.2. A topological mapping $T$ of a Riemann surface $R$ onto another Riemann surface $R^{\prime}$ is a direct or indirect conformal mapping if and

8) See also Bers [3].

9) This is a direct consequence of Mori's Lemma 4 in [14] and Yüjobob's Theorem 4 in [34]. As for dilatations, we have $K^{*}(T) \leqq K(T) \leqq \exp \left(\pi K^{*}(T)\right)$. 
only if $K^{*}(T)=1$.

Remark. We may restate Theorems II. 1 and II. 2 as follows: there exists a finite real-valued increasing function $r(x)$ defined for $1 \leqq x$ such that $r(1)=1$ and $\lim _{x \rightarrow \infty} r(x)=\infty$ and $K^{*}(T) \leqq K(T) \leqq r\left(K^{*}(T)\right)$.

3. $M$-mapping. Let $R$ be a Riemann surface and $M(R)$ be the Royden's algebra associated with $R$, i.e., the algebra of all complex-valued bounded functions on $R$ which are absolutely continuous in the sense of Tonelli and have finite Dirichlet integrals over $R$. The algebra $M(R)$ can be normed

$$
\|f\|=\sup _{R}|f|+\sqrt{D[f]},
$$

where $D[f]$ denotes the Dirichlet integral $\iint_{R}|\operatorname{grad} f|^{2} d x d y$ of $f$ over $R$. Then $M(R)$ is complete with respect to this norm and the subalgebra $M^{1}(R)$ is dense in $M(R)$, where $M^{1}(R)$ denotes the totality of functions in $M(R)$ which are continuously differentiable (cf. Nakai [18]). We denote by $M_{0}(R)$ the subalgebra of $M(R)$ consisting of all members in $M(R)$ which have compact carriers, i.e., $f$ belongs to $M_{0}(R)$ if and only if $f$ belongs to $M(R)$ and vanishes outside a compact set.

A topological mapping $T$ of $R$ onto $R^{\prime}$ is said to be an M-mapping if $T$ induces an algebraic isomorphism of $M_{0}(R)$ onto $M_{0}\left(R^{\prime}\right)$, i.e., $f$ belongs to $M_{0}(R)$ if and only if the composite function $f \circ T^{-1}$ belongs to $M_{0}\left(R^{\prime}\right)$. For an $M$ mapping of $R$ onto $R^{\prime}$, we define the norm of $T$ by

$$
\|T\|=\inf \left\{\lambda ; \lambda^{-1}\|f\| \leqq\left\|f \circ T^{-1}\right\| \leqq \lambda\left\|_{i}\right\|, \text { for all } f \text { in } M_{0}(R)\right\} .
$$

Clearly $1 \leqq\|T\| \leqq \infty$. The aim of the present chapter is to prove the following:

Theorem II.3. An M-mapping $T$ of a Riemann surface $R$ onto another Riemann surface $R^{\prime}$ with finite norm $\|T\|$ is a quasiconformal mapping with the maximal dilation $K(T) \leqq r\left(\|T\|^{2}\right)$. In particular, $T$ is a direct or an indirect conformal mapping if and only if $\|T\|=1$.

4. Fundamental functions. Recall that $\mathbf{A}$ denotes the totality of annuli $A$ on $R$ satisfying the following two conditions:

(A. 1) $A$ is a domain contained in a simply connected domain $D_{A}$ on $R$;

(A.2) the boundary $\partial A$ of $A$ consists of two closed Jordan curves $C_{0}$ and $C_{1}$. 
Assume that the domain $\left(C_{0}\right)$ in $D_{A}$ surrounded by $C_{0}$ contains $C_{1}$. Now we consider the function $f_{A}(p)$ on $R$ defined by the following manner:

$$
f_{A}(p)=\left\{\begin{array}{l}
0, \text { if } p \text { belongs to } R-\left(C_{0}\right) ; \\
w\left(p ; A, C_{1}\right), \text { if } p \text { belongs to } A ; \\
1, \text { if } p \text { belongs to the closure of }\left(C_{1}\right),
\end{array}\right.
$$

where $w\left(p ; A, C_{1}\right)$ denotes the harmonic measure of $C_{1}$ with respect to $A$. Now we prove that $f_{A}$ belongs to $M(R)$ and $D\left[f_{A}\right]=\iint_{A}\left|\operatorname{gard} f_{A}\right|^{2} d x d y$. For the aim, we select a decreasing sequence $\left\{\varepsilon_{n}\right\}$ of positive numbers tending to zero such that $\varepsilon_{n}<1-\varepsilon_{n}$ and the level curves $C_{0, n}=\left\{p ; w\left(p ; A, C_{1}\right)=\varepsilon_{n}\right\}$ and $C_{1, n}=\left\{p ; w\left(p ; A, C_{1}\right)=1-\varepsilon_{n}\right\}$ are simple closed analytic curves. We set

$$
f_{n}(p)=\left\{\begin{array}{l}
\varepsilon_{n}, \text { if } p \text { belongs to } R-\left(C_{0, n}\right) ; \\
w\left(p, A, C_{1}\right), \text { if } p \text { belongs to }\left(C_{0, n}\right)-\text { the closure of }\left(C_{1, n}\right) ; \\
1-\varepsilon_{n}, \text { if } p \text { belongs to the closure of }\left(C_{1, n}\right) .
\end{array}\right.
$$

Then $f_{n}(p)$ is clearly in $M(R)$. Moreover, it is obvious that the sequence $\left\{f_{n}\right\}$ converges to $f_{A}$ uniformly and that $\left\{f_{n}\right\}$ is a Cauchy sequence in $M(R)$. Using the completeness of $M(R)$, we conclude that $f_{A}$ belongs to $M(R)$ and that $\left\|f_{n}-f_{A}\right\|$ tends to zero as $n$ tends to infinity. From this it holds that $D\left[f_{A}\right]$ $=\iint_{A}\left|\operatorname{grad} f_{A}\right|^{2} d x d y$.

We say that $f_{A}$, above defined, is a fundamental function with the base $A$. The totality of fundamental functions will be denoted by $M_{f}(R)$, i.e., $M_{f}(R)$ $=\left\{f_{A} ; A\right.$ belongs to $\left.\mathbf{A}\right\}$. If a function $g$ in $M(R)$ coincides with a function $f$ in $M_{f}(R)$ outside the base of $f$, we shall write $\pi g=f$. Concerning this operation, we have the following

LEMMA II. 1. $D[\pi g] \leqq D[g]$ and $\|\pi g\| \leqq\|g\|$.

Proof. For $g$ in $M(R)$, we can select a sequence $\left\{g_{n}\right\}_{n=1}^{\infty}$ in $M^{1}(R)$ such that $\| g_{n}-g_{\|}^{\|}$tends to zero as $n$ tends to infinity and a fortiori $D\left[g_{n}\right]$ tends to $D[g]$. Let $A$ be the base of $f=\pi g$ and $f_{n}$ be the function in $M(R)$ which coincides with $g_{n}$ outside $A$ and harmonic in $A$ with boundary values $g_{n}$ on $\partial A$. By the Dirichlet principle, it holds $D\left[f_{n}\right] \leqq D\left[g_{n}\right]$. As the sequence $\left\{f_{n}\right\}$ converges to $f$ uniformly in $A$ and $\left|\operatorname{grad} f_{n}\right|$ converges to $|\operatorname{grad} f|$ in $A$ by Harnack's theorem, we have $\iint_{A}|\operatorname{grad} f|^{2} d x d y \leqq \lim \iint_{A}\left|\operatorname{grad} f_{n}\right|^{2} d x d y$. Together with $\iint_{R-A}|\operatorname{grad} f|^{2} d x d v=\iiint_{R-A}\left|\operatorname{grad} f_{n}\right|^{2} d x d y$, we get $D[f] \leqq \lim D\left[f_{n}\right]$ 
$\leqq \lim D\left[g_{n}\right]=D[g]$. As $\sup |\pi g| \leqq \sup |g|$, the second inequality holds.

Next we prove two simple lemmas for functions in $M_{f}(R)$ :

Lemma II.2. For any $f$ in $M_{f}(R)$ and for any positive integer $n$, there exist $2^{n}$ functions $f_{1}, f_{2}, \ldots, f_{2^{n}}$ in $M_{f}(R)$ satisfying the following two conditions:

(i) $D\left[f_{k}\right]=2^{n} D[f] \quad\left(k=1,2, \ldots, 2^{n}\right)$;

(ii) the bases $A_{k}$ of $f_{k}$ are mutually disjoint and contained in the base of $A$ of $f$ and $\bar{A}=\bar{A}_{1} \cup \bar{A}_{2} \cup \cdots \cup \bar{A}_{2^{n}}$, where the bar denotes the closure.

Proof. The general case is easily reduced to the case $n=1$, so we shall prove the lemma under the assumption $n=1$. We define two fundamental functions $f_{1}(p)$ and $f_{2}(p)$ on $R$ as follows:

$$
\begin{aligned}
& f_{1}(p)= \begin{cases}1 & (1 / 2 \leqq f(p)) ; \\
2 f(p) & (0<f(p)<1 / 2) ; \\
0 & (f(p) \leqq 0)\end{cases} \\
& f_{2}(p)= \begin{cases}1 & (1 \leqq f(p)) ; \\
2 f(p)-1 & (1 / 2<f(p)<1) \\
0 & (f(p) \leqq 1 / 2)\end{cases}
\end{aligned}
$$

Then the bases $A_{1}$ and $A_{2}$ of $f_{1}$ and $f_{2}$ are represented by

$$
A_{1}=\{p ; 0<f(p)<1 / 2\}, \quad A_{2}=\{p ; 1 / 2<f(p)<1\} .
$$

Evidently (ii) is satisfied. As we have $\left|\operatorname{grad} f_{k}\right|^{2}=4|\operatorname{grad} f|^{2}$ on $A_{k}(k=1,2)$, it holds that

$$
4 D[f]=4 D_{A_{1}}[f]+4 D_{A_{3}}[f]=D\left[f_{1}\right]+D\left[f_{2}\right],
$$

where $D_{A_{k}}$ denotes the dirichlet integral over $A_{k}$. On the other hand, by using Green's formula, we get

$$
D\left[f_{1}\right]=2 D[f] .
$$

From the above two equalities, it follows $D\left[f_{k}\right]=2 D[f](k=1,2)$, i.e., the condition ( $i$ ) in lemma is satisfied.

Lemma II.3. Let $f$ and $f_{k}(k=1,2, \ldots, n)$ are functions in $M_{f}(R)$ such that the bases $A_{k}$ of $f_{k}$ are mutually disjoint and contained in the base $A$ of $f$ and $\ddot{A}=\bar{A}_{1} \cup A_{2} \cup \cdots \cup \bar{A}_{n}$. Then the following inequality holds:

$$
1 / D[f] \geqq \sum_{k=1}^{n} 1 / D\left[f_{k}\right] \text {. }
$$


Proof. We may assume $n=2$, for the general case is easily reduced to this simple case. We define the function $g(p)$ in $M(R)$ on $R$ as followa:

$$
g(p)= \begin{cases}f_{1}(p) & \left(f_{1}(p)<1\right) \\ \lambda & \left(f_{1}(p)=1, f_{2}(p)=0\right) \\ (1-\lambda)\left(f_{2}(p)+\lambda(1-\lambda)^{-1}\right) & \left(f_{2}(p)>0\right),\end{cases}
$$

where $\lambda$ is an arbitrary number in the interval $0 \leqq \lambda \leqq 1$. Then $g=f$ on $R-A$ and so $\pi g$ can be defined and $f=\pi g$. By Lemma II. 1 , we have

$$
D[f]=D[\pi g] \leqq D[g] .
$$

As $|\operatorname{grad} g|^{2}=\lambda^{2}\left|\operatorname{grad} f_{1}\right|^{2}$ on $\{p ; g(p)<\lambda\}$ and $|\operatorname{grad} g|^{2}=(1-\lambda)^{2}\left|\operatorname{grad} f_{2}\right|^{2}$ on $\{p ; g(p)>\lambda\}$, we have

$$
\begin{aligned}
D[g] & =\iint_{0<g<\lambda}|\operatorname{grad} g|^{2} d x d y+\iint_{\lambda<g<1} \mid \operatorname{grad} g_{i}^{2} d x d y \\
& =\lambda^{2} \iint_{0<f_{1}<1}\left|\operatorname{grad} f_{1}\right|^{2} d x d y+(1-\lambda)^{2} \iint_{0<f_{2}<1}\left|\operatorname{grad} f_{2}\right|^{2} d x d y \\
& =\lambda^{2} D\left[f_{1}\right]+(1-\lambda)^{2} D\left[f_{2}\right] .
\end{aligned}
$$

Thus we get

$$
D[f] \leqq \lambda^{2} D\left[f_{1}\right]+(1-\lambda)^{2} D\left[f_{2}\right] .
$$

In particular, if we put $\lambda=D\left[f_{2}\right]\left(D\left[f_{1}\right]+D\left[f_{2}\right]\right)^{-1}$, then we see that

$$
1 / D[f] \geqq 1 / D\left[f_{1}\right]+1 / D\left[f_{2}\right],
$$

which proves the lemma.

5. Now we consider the relation between the norm $\|T\|_{1}$ and the quantity $K^{*}(T)$ for an $M$-mapping $T$ of $R$ onto $R^{\prime}$. The following plays an essential role in the proof of Theorem II. 3.

Lemma II. 4. For any M-mapping $T$ of $R$ onto $R^{\prime}$, it holds $K^{*}(T) \leqq T_{\|^{\prime 2}}$.

Proof. If $\|T\|=\infty$, nothing is left to be proved. So we may assume that the norm of $T$ is finite. Let $f$ be in $M(R)$. Instead of $f \circ T^{-1}$, we shall write $f^{\prime}$. Let $A$ belong to $\mathbf{A}$. If $f$ is in $M_{f}(R)$ and if the base of $f$ is $A$, then we have

$$
\bmod A=2 \pi / D[f] .
$$

From this we have 
$\sqrt{ } K^{*}(T)=\inf \left\{\lambda ; \lambda^{-1} \sqrt{ } D[f] \leqq \sqrt{ } D\left[\pi f^{\prime}\right\rfloor \leqq \lambda \sqrt{ } D[f]\right.$ for all $f$ in $\left.M_{f}(R)\right\}$.

If we set

$$
\begin{aligned}
& a=\sup \left\{\sqrt{D\left[\pi f^{\prime}\right]} / \sqrt{ } D[f] ; f \text { belongs to } M_{f}(R)\right\}, \\
& a^{\prime}=\sup \left\{\sqrt{D[f]} / \sqrt{D\left[\pi f^{\prime}\right]} ; f \text { belongs to } M_{f}(R)\right\} \text {, }
\end{aligned}
$$

then we see that $\sqrt{ } K^{*}(T)=\max \left(a, a^{\prime}\right)$. So, if we can show that $a, a^{\prime} \leqq\|T\|$, then the proof is complete. We have only to show that $a \leqq\|T\|$, since $a^{\prime} \leqq\|T\|$ can be verified by the similar argument. For the purpose, we set

$$
a_{n}=\sup \left\{\sqrt{D\left[\pi f^{\prime}\right]} / \sqrt{D[f]} ; \text { for all } f \text { in } M_{f, n}(R)\right\} \quad(n=1,2, \ldots),
$$

where $M_{f, n}(R)=\left\{f ; f\right.$ belongs to $M_{f}(R)$ and $\left.\sqrt{D[f]} \geqq n\right\}$. From the definition it is clear that the sequence $\left\{a_{n}\right\}$ is monotone non-increasing. First we show that

(I) for any positive number $s$, there exists a positive integer $N$ depending on $\varepsilon$ such that

$$
a_{.}<\|T\|+\varepsilon .
$$

Contrary to the assertion, we assume the existence of a positive number $\varepsilon$ such that $\|T\|+\varepsilon \leqq a_{n}$ for all $n=1,2, \ldots$ Then we can choose two positive numbers $b$ and $c$ such that

$$
a_{n}>b>c>\|T\| \geqq 1
$$

for all positive integers $n$. By the definition of $a_{n}$, we can find an elemant $f^{(n)}$ in $M_{f, n}(R)$ for each $n=1,2, \ldots$ such that

$$
\sqrt{ } D\left[\pi f^{(n) \prime}\right]>b \sqrt{D\left[f^{(n)}\right]} .
$$

Now we choose an integer $n$ so large as to satisfy the inequality $n>c(b-c)^{-1}$ Then we have $\sqrt{ } D\left[f^{(n)}\right]>n>c(b-c)^{-1}$ or

$$
(b-c) \sqrt{D\left[f^{(n)}\right]>c .}
$$

We proceed to compute as follows: using Lemma 1, we have

$$
\begin{aligned}
\left\|f^{(n) \prime}\right\| & \geqq\left\|\pi f^{(n) \prime}\right\|>\sqrt{D\left[\pi f^{(n) \prime}\right]} \\
& \left.>b \sqrt{ } D\left[f^{(n)}\right]=(b-c) \sqrt{D\left[f^{(n)}\right.}\right]+c \sqrt{D}\left[f^{(n)}\right] \\
& >c+c \sqrt{D\left[f^{(n)}\right]}=c\left\|f^{(n)}\right\|,
\end{aligned}
$$

that is, 


$$
c\left\|f^{(n)}\right\|<\left\|f^{(n)}\right\|^{\prime}
$$

On the other hand, $\left\|f^{(n)}\right\| \leqq\|T\|\left\|f^{(n)}\right\|$. From the above inequality, we get $c\left\|f^{(n)}\right\|<\|T\|\left\|f^{(n)}\right\|$ or $c<\|T\|$, which contradicts the definition of $c$. Thus we have proved (I). Next we show that

(II) for any positive integer $m$ and for any $f$ in $M_{f}(R)$, it holds

$$
\sqrt{D\left[\pi f^{\prime}\right]} \leqq a_{m} \sqrt{D[f]} .
$$

To see this, we choose a sufficiently large positive integer $n$ such that $2^{n} D[f]>m^{2}$. By Lemma II. 2 , we can choose $2^{n}$ functions $f_{k}(k=1,2, \ldots$, $\left.2^{n}\right)$ in $M_{f}(R)$ such that

$$
D\left[f_{k}\right]=2^{n} D[f]
$$

and the bases of $f$ and $f_{k}$ satisfy the condition (ii) in Lemma II.2. By the definition of $n, D\left[f_{k}\right]=2^{n} D[f]>m$ or $f_{k}$ is in $M_{f, m}(R) \quad\left(k=1,2, \ldots, 2^{n}\right)$. Then, by the definition of $a_{m}$, we have

$$
\sqrt{D\left[\pi f_{k}^{\prime}\right]} \leqq a_{m} \sqrt{D\left[f_{k}\right]} \text { or } 1 / D\left[f_{k}\right] \leqq a_{m l}^{2} / D\left[\pi f_{k}^{\prime}\right] \text {. }
$$

Thus we get

$$
1 / D[f]=2^{n} / 2^{n} D[f]=\sum_{k=1}^{2^{n}} 1 / D\left[f_{k}\right] \leqq a_{m}^{2} \sum_{k=1}^{2^{n}} 1 / D\left[\pi f_{k}^{\prime}\right]
$$

On the other hand, $\pi f^{\prime}$ and $\pi f_{k}^{\prime}\left(k=1,2, \ldots, 2^{n}\right)$ satisfy the assumption of Lemma III. 3, so it holds

$$
\sum_{k=1}^{2^{\prime n}} 1 / D\left[\pi f_{k}^{\prime}\right] \leqq 1 / D\left[\pi f^{\prime}\right]
$$

Combining the above two inequalities, we get

$$
\sqrt{D}\left[\pi f^{\prime}\right] \leqq a_{m} \sqrt{D[f]} .
$$

Finally we shall prove $a \leqq\|T\|$. To this end, we choose an arbitrary positive number $\varepsilon$. Then, by (I), we get a positive integer $N$ such that

$$
a_{\mathrm{N}}<\|T\|+\varepsilon .
$$

For any $f$ in $M_{f}(R)$, it holds that $\sqrt{ } D\left[\pi f^{\prime}\right] \leqq a_{x} \sqrt{D[f] \leqq}\left(\|T\|_{1}+\varepsilon\right) \sqrt{D[f]}$ by (II). As $\varepsilon$ is indpendent of $\|T\|$ and $f$, letting $\varepsilon$ tend to 0 , we find that

$$
\sqrt{D\left[\pi f^{\prime}\right]} \leqq\|T\| \sqrt{ } D[f]
$$


for all $f$ in $M_{f}(R)$, which shows that $a \leqq\|T\|$. This completes the proof of Lemma II. 3.

6. Now the proof of Theorem II. 3 is immediate. By Lemma II. 3 and by Remark in II. 2 , we have

$$
1 \leqq K^{*}(T) \leqq\|T\|^{2} \leqq K(T)^{10)} \leqq r\left(K^{*}(T)\right) \leqq r\left(\|T\|^{2}\right) .
$$

From this we get Theorem II. 3.

\section{Algebraic criterion on quasiconformal equivalence}

1. If there exists a topological mapping $T$ of a Riemann surface $R$ onto another Riemann surface $R^{\prime}$, then we say that $R$ and $R^{\prime}$ are topologically equivalent and we write $R \sim R^{\prime}$. Moreover, if $T$ is quasiconformal, then $R$ and $R^{\prime}$ are said to be quasiconformally equivalent and we denote it by $R \simeq R^{\prime}$. In particular if the maximal dilatation of $T$ is 1 , i.e., if $T$ is a direct or indirect conformal mapping, then we say that $R$ and $R^{\prime}$ are conformally equivalent and we denote the fact by $R \cong R^{\prime}$.

As for the topological equivalence, results of Hewitt [8] implies that $R \sim R^{\prime}$ if and only if $C(R) \cong C\left(R^{\prime}\right){ }^{11)} \quad$ Sometimes it is more convinient to use $B(R)$ than to use $C(R)$, since the normed ring theory can be applied to $B(R)$ and since $C(R) \cong C\left(R^{\prime}\right)$ implies $B(R) \cong B\left(R^{\prime}\right)$ but the converse is not true. In this point of view, we may state the following fact: $R \sim R^{\prime}$ if and only if $B(R)$ $\cong B\left(R^{\prime}\right)$. This is a direct consequence of Theorem 1.1 by considering the property $P$ in the theorem as the property $B$.

For compact surfaces $R$ and $R^{\prime}$, it is well known that $R \simeq R^{\prime} \Leftrightarrow R \sim R^{\prime}$ $\Longleftrightarrow B(R) \cong B\left(R^{\prime}\right) \Longleftrightarrow C(R) \cong C\left(R^{\prime}\right) \Longleftrightarrow g(R)=g\left(R^{\prime}\right)$, where $g(R)$ denotes the genus of $R$. But for open surfaces, none of the latter four determines the quasiconformal equivalence $R \simeq R^{\prime}$.

2. The main theorems. As for the quasiconformal (or conformal) equivalence, we can state the following purely algebraic (or normed algebraic) criterion of the quasiconformal (or conformal) equivalence using the Royden's.algebra,

10) As for $\|T\|^{2} \leqq K(T)$, see [18].

11) $C(R)$ (resp. $B(R)$ ) denotes the algebra of all complex valued continuous (resp. bounded continuous) functions on $R$. For algebras $A$ and $A^{\prime}, A \cong A^{\prime}$ means that $A$ is. isomorphic to $A^{\prime}$ as algebras over the complex number field. 
which are the main object of this paper.

THEOREM III.1. Two Riemann surfaces $R$ and $R^{\prime}$ are quasiconformally equivalent if and only if their Royden's algebras $M(R)$ and $M\left(R^{\prime}\right)$ are isomorphic as algebras.

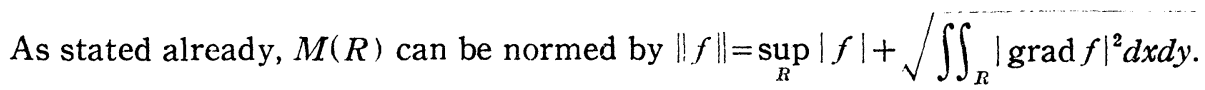
With this norm, $M(R)$ is a normed algebra. Then we can prove

TheOREM III.2. Two Riemann surfaces $R$ and $R^{\prime}$ are conformally equivalent if and only if their Royden's algebras $M(R)$ and $M\left(R^{\prime}\right)$ are isomorphic as normed algebras. $^{12)}$

3. Theorems III. 1 and III. 2 follow from the following more precise facts. Let $Q\left(R, R^{\prime}\right)$ be the totality of quasiconformal mappings of $R$ onto $R^{\prime}$ and $I\left(R, R^{\prime}\right)$ be the totality of algebraic isomorphisms of $M(R)$ onto $M\left(R^{\prime}\right)$. For $\sigma$ belonging to $I\left(R, R^{\prime}\right)$, we put $\|\sigma\|=\inf \left\{\lambda ; \lambda^{-1}\|f\| \leqq\left\|f^{\sigma}\right\| \leqq \lambda\|f\|\right\}$. Clearly $1 \leqq\|\sigma\| \leqq \infty$. Then Theorems III. 1 and III. 2 are direct consequences of the following

THEOREM III.3. There exists a one-to-one correspondence $\sigma \leftrightarrow T$ between $I\left(R, R^{\prime}\right)$ and $Q\left(R, R^{\prime}\right)$ satisfying (i) $f^{\circ}=f \circ T^{-1}$ for $f$ in $M(R)$ and (ii) $1 \leqq K^{*}(T) \leqq\left\|_{\sigma}\right\|^{2} \leqq K(T)$

4. Property M. For the proof of Theorem III.3, we apply the general Theorem I. 1. For the aim, we introduce a property $M$ on continuous functions. Let $M$ be a subfamily of $C=\{(f, S)\}$ satisfying the following four conditions : a pair $(f, S)$ belongs to $M$ if and only if

(M. 0) $S$ is an open subset of a Riemann surface $R_{S}$;

(M. 1) the function $f$ is a.c. $T$ on $S$;

(M. 2) $f$ is bounded on $S$ in the absolute value;

(M. 3) the Dirichlet integral $\iint_{S}|\operatorname{grad} f|^{2} d x d y$ of $f$ over $S$ is finite.

Now we put $\|(f, S)\|_{i}=\left.\left|f\left\|_{S}=\right\|_{i} f \|_{i}=\sup _{R}\right| f\left|+\iiint_{R}\right| \operatorname{grad} f\right|^{2} d x d y$ for a pair $(f, S)$ belonging to $M$. We shall show that $M$ is an admissible property and that any Riemann surface belongs to $\mathbf{S}_{m}$. The following is evident:

12) This means that $M(R)$ and $M\left(R^{\prime}\right)$ are isomorphic as algebras and that the norms of corresponding elements by this isomorphism are coincident. 
Lemma III.1. The property $M$ satisfies the conditions (P.1), (P.2), (P.3), (P.4), (P.5) and (P.6).

5. Next we have to show that $M$ satisfies (P.7). For our purpose, the following lemma is very important:

Lemma III.2. Let $S$ and $S^{\prime}$ satisfy (M.0) and let $\sigma$ be an algebraic isomorphism of $M(S)$ onto $M\left(S^{\prime}\right)$. Then the following holds: $1 \leqq\|\sigma\|<\infty$, where $\|\sigma\|=\inf \left\{\lambda ; \lambda^{-1}\|f\| \leqq\left\|f^{\sigma}\right\| \leqq \lambda\|f\|\right.$ for all $f$ in $\left.M(S)\right\}$.

Proof. Consider $M(S)$ to be a normed algebra with the norm $\|f\|$. Then $M(S)$ is complete with respect to this norm $\|f\|$ (cf. [18]). ${ }^{13)}$ As $\left\|f^{n}\right\|_{1}^{1 / n}$ $\geqq \sup _{R}|f|$, we can conclude that $\lim \left\|f^{n}\right\|^{1 / n}=0$ if and only if $f=0$, which shows $M(S)$ has no generalized nilpotent. Since $M(S)$ is commutative, this is equivalent to that $M(\mathrm{~S})$ is semi-simple. Thus $\sigma$ is an algebraic isomorphism of a commutative semi-simple Banach algebra $M(S)$ onto such a $M\left(S^{\prime}\right)$. Then by the Gelfand theorem [6], ${ }^{14)}$ we have the bicontinuity of $\sigma$ with respect to the norm topology. From this we get the finiteness of $\sigma$.

Next we prove

Leмma III.3. $M$ is an admissible property on continuous functions.

Proof. By virtue of Lemma III.1, we have only to show that $M$ satisfies (P.7). Let $\sigma$ be an algebraic isomorphism of $M(S)$ onto $M\left(S^{\prime}\right)$ and let $U$ and $U^{\prime}$ be two open subsets of $S$ and $S^{\prime}$, respectively, and let $T$ be a topological mapping of $U$ onto $U^{\prime}$ such that $f^{\sigma}(T p)=f(p)$ on $U$. We show that $T$ has property $M$, i.e., $\left(g \circ T^{-1}, U^{\prime}\right)$ belongs to $M$ if and only if $(g, U)$ belongs to $M$. As the argument is quite similar, we have only to show that $\left(g \circ T^{-1}, U^{\prime}\right)$ belongs to $M$ if $(g, U)$ does so.

Let $U=\sum U_{n}$ be the decomposition of $U$ into components and $U^{\prime}=\sum U_{n}^{\prime}$ be the corresponding one for $U^{\prime}$, i.e., $T U_{n}=U_{n}^{\prime}$. We denote by $T_{n}$ the restriction of $T$ on $U_{n}$. Then $T_{n}$ is a homeomorphism of $U_{n}$ onto $U_{n}^{\prime}$. Then it is clear that $f \rightarrow f^{s}=f \circ T_{n}^{-1}$ is an algebraic isomorphism between $M_{0}\left(U_{n}\right)$ and $M_{0}\left(U_{n}^{\prime}\right)$. The norm $\left\|T_{n}\right\|$ of $T_{n}$ defined in II.3 is not larger than $\left\|\sigma_{0}\right\|$ which is finite by Lemma III.2. By Theorem II.3, $T_{n}$ is a quasiconformal mapping with

13) In [18], $S$ is assumed to be connected, but the proof stated there had make no use of the connectedness of $S$.

14) See also Loomis [13]. 
the dilatation not larger than $r\left(\|\sigma\|^{2}\right)$.

Let $(g, U)$ be in $M$. For simplicity, we put $K=r\left(\|\sigma\|^{2}\right), g^{\prime}=g \circ T^{-1}$ and $g_{n}^{\prime}=g \circ T_{n}^{-1}$. It is clear that $\left(g^{\prime}, U^{\prime}\right)$ satisfies (M.0) and (M.2). By Theorem 2 in [18], we see that $g_{n}^{\prime}$ is a.c.T and $D_{U^{\prime} n}\left[g_{n}^{\prime}\right] \leqq K D_{U_{n}}[g]$. From this, $g^{\prime}$ is a.c. $T$ and $D_{D^{\prime}}\left[g^{\prime}\right]=\sum D_{U^{\prime}{ }_{n}}\left[g_{n}^{\prime}\right] \leqq \sum K D_{U_{n}}[g]=K D_{U}[g]$. Thus $\left(g^{\prime}, U\right)$ satisfies (M.1) and (M.3).

6. Now we prove the following

Lemma III. 4. Any open subset $S$ of a Riemann surface belongs to $\mathbf{S}_{M}$.

Proof. In virtue of (P.1), (P.2) and (P.6), we have only to show that the center $z=0$ of the disc $U:|z|<1 / 2$ is not $M$-removable. Consider the function

$$
f(z)=\sin \left(\log \left(\log \left(|z|^{-1}\right)\right)\right)
$$

defined on $U_{0}=U-\{0\}$. Clearly $\left(f, U_{0}\right)$ satisfies (M.0), (M.1) and (M.2).

Further, we have

$$
\begin{aligned}
\iint_{U_{0}}|\operatorname{grad} f(z)|^{2} d x d y & =\int_{0}^{2 \pi} \int_{0}^{1 / 2}\left(|\partial f / \partial r|^{2}+|\partial f / \partial \theta|^{2} / r^{2}\right) r d r d \theta \\
& =2 \pi \int_{0}^{1 / 2} \cos ^{2}\left(\log \left(\log r^{-1}\right)\right) r^{-1}(\log r)^{-2} d r \\
& \leqq 2 \pi \int_{0}^{1 / 2} r^{-1}(\log r)^{-2} d r=2 \pi(\log 2)^{-1}
\end{aligned}
$$

which shows that $\left(f, U_{0}\right)$ satisfies (M.3). Thus $\left(f, U_{0}\right)$ belongs to $M$.

Consider the cluster set $C_{v_{0}}(f, 0)$ of $f$ at 0 . It is easy to see that $C_{U_{0}}(f, 0)$ is the closed interval $[-1,1]$. Hence there exists no pair $(g, U)$ in $M$ such that $g(z)=f(z)$ on $U_{0}$. Thus 0 is not $M$-removable.

7. Proof of Theorem III.3. We define a mapping $\Sigma: T \rightarrow T^{\Sigma}=\sigma$ of $Q\left(R, R^{\prime}\right)$ into $I\left(R, R^{\prime}\right)$ as follows: $f^{\sigma}=f \circ T^{-1}$, which is nothing but (i) in the theorem. By results in [18] and [19], this is well defined and (ii) in our theorem is satisfied. Moreover, it is clear that $\Sigma$ is one-to one. So we have only to show that $\Sigma$ is an onto mapping. Let $\sigma$ be an arbitrary element in $I\left(R, R^{\prime}\right)$. Then, by Theorem I.1, Lemma III. 3 and Lemma III.4, we find a topological mapping of $R$ onto $R^{\prime}$ such that $f^{\jmath}=f \circ T^{-1}$. Of course, $f \rightarrow f \circ T^{-1}$ induces an algebraic isomorphism of $M_{0}(R)$ onto $M_{0}\left(R^{\prime}\right)$ and the norm $\|T\|$ of $T$ is not larger than $\|\sigma\|$ which is finite by Lemma III.2. Thus, by Theorem 
II. 3, we see that $T$ belongs to $Q\left(R, R^{\prime}\right)$ and $T^{\Sigma}=\sigma$. This shows that $\Sigma$ is onto.

This completes the proof of Theorem III. 3.

8. As an immediate consequence of Theorem 2 in [18] and Theorem III.3, we get the following

THEOREM III.4. An algebraic isomorphism of $M(R)$ onto $M\left(R^{\prime}\right)$ is bicontinous with respect to the following topologies in $M(R)$ and $M\left(R^{\prime}\right)$ :

(i ) Norm-topology: $\left\|f_{n}^{\sigma}\right\| \rightarrow 0$ if and only if $\left\|f_{n}\right\| \rightarrow 0$;

(ii) Uniform topology: $\sup _{R^{\prime}}\left|f_{n}^{\sigma}\right|=\sup _{R}\left|f_{n}\right|$;

(iii) D-topology: $\quad D\left[f_{n}^{\sigma}\right] \rightarrow 0$ if and only if $D\left[f_{n}\right] \rightarrow 0$;

(iv) B-topology: $\quad\left\{\left|f_{n}^{\{}\right|\right\}$is bounded and $f_{n}^{\{} \rightarrow 0$ uniformly on each compact set of $R^{\prime}$ if and only if $\left\{\left|f_{n}\right|\right\}$ is bounded and $f_{n} \rightarrow 0$ uniformly on each compact set of $R$;

( v) BD-topology: $f_{n}^{a} \rightarrow 0$ (in $B$ and D-topology) if and only if $f_{n} \rightarrow 0$ (in $B$ and D-topology).

9. Differentiable function subalgebras of $\boldsymbol{M}(\boldsymbol{R})$. Next we consider the subalgebra $M^{n}(R) \quad(n=1,2, \ldots, \infty)$ of $M(R)$ consisting of all the elements in $M(R)$ which are $n$-fold continuously differentiable; i.e., a complex-valued function $f$ on $R$ belongs to $M^{n}(R)$ if and only if

( $\mathbf{M}^{n}$. 1) $f$ belongs to the class $C^{n}$;

( $\mathbf{M}^{n}$.2) $f$ is bounded on $R$ in the absolute value;

$\left(\mathbf{M}^{n} .3\right)$ Dirichlet integral $D[f]$ of $f$ over $R$ is finite.

For convenience we put $M^{0}(R)=M(R)$. As a subalgebra of $M(R), M^{n}(R)$ can be normed by the norm of $M(R)$, i.e., $\|f\|=\sup _{R}|f|+\sqrt{D[f]}$. Needless to say, $M^{n}(R)$ is not complete with respect to this norm in the case $n \geqq 1$ but we have proved, as already stated and used, in [18] the following ${ }^{15)}$

Lemma III.5. For each $n=1,2, \ldots, \infty, M^{n}(R)$ is dense in $M(R)$.

10. If there exists a $C^{n}$-quasiconformal (resp. conformal) mapping $T$ of $R$ onto $R^{\prime}$, then the correspondence $f \rightarrow f^{\sigma}=f \circ T^{-1}$ induces an algebraic (resp. normed algebraic) isomorphism of $M^{n}(R)$ onto $M^{n}\left(R^{\prime}\right)$. This leads us naturally

15) In [18], the case $n=\infty$ is not treated. But a similar method, by using molifier instead of mean-value integral of Rad6, can be used to include the case $n=\infty$. 
to study the converse problem: under the assumption that there exists an algebraic (resp. normed algebraic) isomorphism of $M^{\prime \prime}(R)$ onto $M^{n}\left(R^{\prime}\right)$, can we conclude the existence of a $C^{n}$-quasiconformal (resp. conformal) mapping of $R$ onto $R^{\prime}$ which induces a given isomorphism? The answer is affirmative. To state our result, we introduce two notations: $Q^{n}\left(R, R^{\prime}\right)$ denotes the totality of $C^{n}$-quasiconformal mappings of $R$ onto $R^{\prime}(n=1,2, \ldots, \infty)$ and $I^{n}\left(R, R^{\prime}\right)$ denotes the totality of algebraic isomorphisms of $M^{n}(R)$ onto $M^{n}\left(R^{\prime}\right) \quad(n=1$, $2, \ldots, \infty)$. For convenience we put $Q\left(R, R^{\prime}\right)=Q^{0}\left(R, R^{\prime}\right)$ and $I\left(R, R^{\prime}\right)$ $=I^{0}\left(R, R^{\prime}\right)$. Then we can prove

THEOREM III.5. There exists a one-to-one correspondence $\sigma \leftrightarrow T$ between $I^{n}\left(R, R^{\prime}\right)$ and $Q^{n}\left(R, R^{\prime}\right)$ satisfying (i) $f^{\sigma}=f \circ T^{-1}$ for all $f$ in $M^{n}(R)$ and (ii) $1 \leqq K^{*}(T)\|\sigma\|^{2} \leqq K(T)$.

11. In the case $n=0$, this theorem reduces to Theorem III.3. Thus we may assume $n \geqq 1$. In the case $n=0$, the completeness of $M(R)$ has played a very important role (cf. Lemma III. 2 ). In the case $n \geqq 1$, we essentially use the continuity of the dilatation at a point in place of the completeness.

As before we define a mapping $\Sigma_{n}: T \rightarrow T^{\Sigma_{n}}=\sigma$ of $Q^{n}\left(R, R^{\prime}\right)$ into $I^{n}\left(R, R^{\prime}\right)$ as follows: $f^{3}=f \circ T^{-1}$ for $f$ in $M^{n}(R)$, which is (i) in the present theorem. Clearly this is well defined and one-to-one and satisfies (ii). We shall prove that this is onto.

12. For the aim, we define a property $M^{n}$ by the following: a pair $(f, S)$ in $C=\{(f, S)\}$ belongs to $M^{n}$ if and only if $S$ is an open subset of a Riemann surface $R$ and $f$ is bounded $C^{n}$-function on $S$ with finite Dirichlet integral over S. Then it is easy to see that $M^{n}$ satisfies (P.1), (P.2), (P.3), (P.4), (P.5) and (P.6). Moreover, any open subset of a Riemann surface belongs to $\mathbf{S}_{1}$. For the proof of this, the proof of Lemma III. 4 can be applied to this case without any change. In order to apply Theorem I. 1, we now have only to show that the property $M^{n}$ satisfies (P.7).

Let $\sigma$ be an algebraic isomorphism of $M^{n}(S)$ onto $M^{n}\left(S^{\prime}\right)$. Assume that $\sigma$ is induced by $T$ locally on $U$ and $U^{\prime}$, i.e., $T$ is a topological mapping of an open subset $U$ of $S$ onto an open subset $U^{\prime}$ of $S^{\prime}$ such that $f^{\sigma}(T p)=f(p)$ on $U$ for all $f$ in $M(S)$. Let $U=\sum U_{r}$ be a decomposition of $U$ into components and $U^{\prime}=\sum U_{r}^{\prime}$ be the corresponding one for $U^{\prime}$ such that $T U_{r}=U_{r}^{\prime}$. 
First we prove that $T$ is a $C^{n}$-quasiconformal mapping of $U_{n}$ onto $U_{n}^{\prime}$. It is readily seen that $T$ and $T^{-1}$ are $C^{n}$-mappings. Thus $J(T)=|\partial T / \partial z|^{2}-|\partial T / \partial \bar{z}|^{2}$ vanishes nowhere in $U_{r}$. Now we put

$$
K(T, p)=(|\partial T / \partial z|+|\partial T / \partial \bar{z}|) /|| \partial T / \partial z|-| \partial T / \partial \bar{z}|| \text { at } p,
$$

where $z$ is a local parameter at $p$. Then $K(T, p)$ is continuous with respect to $p$ and $K_{U_{r}}(T)=\sup \left(K(T, p) ; p\right.$ is in $\left.U_{r}\right)$. We have to show that $K_{U_{r}}(T)$ is finite.

Contrary to the assertion, assume that $K_{U_{r}}(T)=\infty$. Then we find a sequence $\left\{p_{m}\right\}$ in $U_{r}$ such that $K\left(T, p_{m}\right)=K\left(T^{-1}, p_{m}^{\prime}\right) \rightarrow \infty \quad(m \rightarrow \infty)$, where $p_{m}^{\prime}=T p_{m}$. Clearly $\left\{p_{m}\right\}$ and $\left\{p_{m}^{\prime}\right\}$ do not cluster in $U_{r}$ and $U_{r}^{\prime}$, respectively.

Now we notice that the following case does not occur: there exists a subsequence $\left\{p_{m^{\prime}}\right\}$ of $\left\{p_{m}\right\}$ such that $\left\{p_{m^{\prime}}\right\}$ and $\left\{p_{m^{\prime}}^{\prime}\right\}$ converge to $p$ and $p^{\prime}$ in the relative boundary of $U_{r}$ and $U_{r}^{\prime}$ with respect to $S$ and $S^{\prime}$ respectively. In fact, if this is not true, then by the remark at the end of Chapter I, we can extend $T$ to be a topological mapping of $V$ onto $V^{\prime}$ such that $\{p\} \cup U_{r} \subset V,\left\{p^{\prime}\right\} \cup U_{r}^{\prime}$ $\subset V^{\prime}$ and $f(T p)=f(p)$ on $V$ for all $f$ in $M^{n}(S)$. From this we can easily see that $T$ is in the class $C^{n}$ in $V$. Choose a compact domain $D$ contained in $V$ with its closure such that $\left\{p, p_{m^{\prime}} ; m=1,2, \ldots\right\}$ is contained in $D$. Then, by the continuity of $K(T, p)$ in the closure of $D, K(T, p)$ is finite in $D$, which contradicts the fact $\lim K\left(T, p_{m^{\prime}}\right)=\infty$.

Now, we may assume that we can choose a sequence $\left\{p_{m}\right\}$ on $U_{r}$, a sequence $\left\{D_{m}\right\}$ of the circular neighborhoods $D_{m}$ of $p_{m}$ satisfying the following

( i ) $\left\{p_{m}\right\}$ does not converge to any point $p$ in the relative boundary of $U_{r}$ with respect to $S$;

(ii) $\lim K\left(T, p_{m}\right)=\infty$;

(iii) the closure of $D_{m}$ is contained in $U_{r} ;{ }^{16)}$

(iv) $D_{m} \cap D_{l}=\phi$ (empty set) if $m \neq l$;

(v) $\widetilde{K}_{D_{m}}(T)=\sup \left\{D\left[\pi\left(f \circ T^{-1}\right)\right] / D[f] ; f\right.$ belongs to $\left.M_{f}\left(D_{m}\right)\right\}>3 m$.

In fact, by the above remark, we may assume (i) and (ii) hold for some $\left\{p_{m}\right\}$. Then (iii) and (iv) are clearly satisfied. Assume that (v) is not satisfied by any subsequence of $\left\{p_{m}\right\}$. Then we have a constant $1<c<\infty$ such that

16) Moreover, we assume that the diameter of $D_{m}$ is smaller than $1 / m$ for some metric on $R$ which induces the topology of $R$. 
$D\left[f \circ T^{-1}\right] \leqq c D[f]$ for all $f$ in $M\left(D_{m}\right) \quad(m=1,2, \ldots)$. In this case, $\left\{p_{m}^{\prime}\right\}$ contains a subsequence which does not converge to any point $p^{\prime}$ in the relative boundary of $U_{r}^{\prime}$ with respect to $S^{\prime}$.

Otherwise, we can find a subsequence of $\left\{p_{m}^{\prime}\right\}$ which converges to a point $p^{\prime}$ in the relative boundary of $U_{r}^{\prime}$. with respect to $S^{\prime}$. For simplicity, we may assume $\left\{p_{m}^{\prime}\right\}$ converges to $p^{\prime}$. Now we choose, for each $m$, an element $\tilde{f}_{m}$ in $M_{f}\left(D_{m}\right)$ such that $\sqrt{ } D\left[\widetilde{f}_{m}\right]<1 / 2^{m}$. By Lemma 1.3 in [18], we can fined an element $f_{m}$ in $M_{0}^{n}\left(D_{m}\right)$ such that $\left\|f_{m}-\widetilde{f}_{m}\right\|<1 / 2^{m}$. We set $f=\sum f_{m}$. Then $\sup _{U r}|f|<3 / 2$ and $\sqrt{D[f]} \leqq \sum_{m} \sqrt{D\left[f_{m}\right]} \leqq \sum\left(\sqrt{D\left[f_{m}\right]}+1 / 2^{m}\right)=2$. Moreover, $f\left(p_{m}\right)=f_{m}\left(p_{m}\right)$ differs from 1 at most $1 / 2^{m}$. Hence $\lim _{m} f\left(p_{m}\right)=1$. On the other hand, we take a point $q_{m}$ on the external boundary of the base of $f_{m}$. Then $f\left(q_{m}\right)=f_{m}\left(q_{m}\right)$ differs from 0 at most $1 / 2^{m}$. Hence $\lim _{m} f\left(q_{m}\right)=0$. Here we remark that both $\left\{p_{m}^{\prime}\right\}$ and $\left\{q_{m}^{\prime}\right\}$ converge to $p^{\prime}$, where $q_{m}^{\prime}=T q_{m}$.

As $f$ vanishes on a neighborhood of the relative boundary of $U_{r}$ with respect to $S$, we may consider that $f$ belongs to $M^{n}(S)$ by defining $f=0$ outside $S$. From $f^{o}(T p)=f(p)$ on $S$ and, hence, on $U_{r}$, we have $f^{\gamma}\left(p_{m}^{\prime}\right)=f\left(p_{m}\right)$ and $f^{\sigma}\left(q_{m}^{\prime}\right)=f\left(q_{m}\right)$. From this we get $f^{\sigma}\left(p^{\prime}\right)=\lim _{m} f^{\sigma}\left(p_{m}^{\prime}\right)=1$ and at the same time $f^{\sigma}\left(p^{\prime}\right)=\lim _{m} f^{\sigma}\left(q_{m}^{\prime}\right)=0$. This contradicts the continuity of $f$ at $p^{\prime}$. Thus we have shown that $\left\{p_{m}^{\prime}\right\}$ satisfies the conditions (i) and (ii) instead of $p_{m}$ since $K\left(T, p_{m}\right)=K\left(T^{-1}, p_{m}^{\prime}\right)$. Putting $D_{m}^{\prime}=T D_{m}$, we see that $\left\{D_{m}^{\prime}\right\}$ satisfies the conditions (iii) and (iv). Next we show that $\lim _{m} \widetilde{K}_{D_{m^{\prime}}}\left(T^{-1}\right)=\infty$, where $\widetilde{K}_{D_{m^{\prime}}}\left(T^{-1}\right)=\sup \left\{D[\pi f] / D\left[f^{\sigma}\right]: f^{\sigma}\right.$ belongs to $\left.M_{f}\left(D_{m}^{\prime}\right)\right\}$. If this is false, we see that $d=\operatorname{Max}\left(K_{D_{m}}(T), K_{D_{m}}\left(T^{-1}\right)\right)$ is finite. Since $K^{*}(T) \leqq d$ and $K(T)$ $\leqq r\left(\left(K^{*}(T)\right)^{2}\right)$, we get that $K(T)$ is finite, which contradicts $K_{U_{r}}(T)=\infty$. Thus we can select the subsequence $\left\{p_{m^{\prime}}^{\prime}\right\}$ of $\left\{p_{m}^{\prime}\right\}$ such that $K_{D_{m^{\prime}}}\left(T^{-1}\right)>3 m$. Hence the conditions (i) $-(\mathrm{v})$ are satisfied by the system $\left\{p_{m^{\prime}}^{\prime}\right\}$ and $\left\{D_{m^{\prime}}^{\prime}\right\}$.

Thus we may consider that $\left\{p_{m}\right\}$ and $\left\{D_{m}\right\}$ satisfy the conditions (i)-(v) (if not so, we may consider $\left\{p_{m^{\prime}}^{\prime}\right\},\left\{D_{m^{\prime}}^{\prime}\right\}$ ).

Now we can find an element $\widetilde{g}_{m}$ in $M_{f}\left(D_{m}\right)$ such that $D\left[\pi\left(\widetilde{g}_{m} \circ T^{-1}\right)\right]$ $>2 m D\left[\widetilde{g}_{m}\right]$ and so

$$
D\left[\widetilde{g}_{m} \circ T^{-1}\right]>2 m D\left[\widetilde{g}_{m}\right]
$$

by the condition (ii). Here we may assume $D\left[\widetilde{g}_{m l}\right]>1$. In fact, by Lemma II. 2 , we can find $2^{l}$ functions $\widetilde{g}_{m}^{k}\left(k=1,2, \ldots, 2^{l}\right)$ in $M_{f}\left(D_{m}\right)$ satisfying (i) 
and (ii) in Lemma II.2. Then, by Lemma II.3,

$$
\begin{aligned}
1 /\left(2^{l} D\left[\widetilde{g}_{m}\right]\right) & >2 m /\left(2^{l} D\left[\pi\left(\widetilde{g}_{m} \circ T^{-1}\right)\right] \geqq 2^{-l} \sum_{R}\left(2 m / D\left[\pi\left(\widetilde{g}_{m}^{k} \circ T^{-1}\right)\right]\right)\right. \\
& \geqq 2^{-l} \sum_{k}\left(2 m / D\left[\widetilde{g}_{m}^{k} \circ T^{-1}\right]\right) .
\end{aligned}
$$

Then, for some $k, 2^{-l} / D\left[\widetilde{g}_{m}\right]>2 m / D\left[\widetilde{g}_{m}^{k} \circ T^{-1}\right]$. So we get $D\left[\widetilde{g}_{m}^{k} \circ T^{-1}\right]$ $>2 m D\left[\widetilde{g}_{m}^{k}\right]$. If we choose $l$ so large as to satisfy $2^{l} D\left[\widetilde{g}_{m}\right]>1$, then we get the required situation.

As $T$ is a quasiconformal mapping of $D_{m}$ onto $D_{m}^{\prime}$, the mapping $f \rightarrow f \circ T^{-1}$ of $M_{0}\left(D_{m}\right)$ onto $M_{0}\left(D_{m}^{\prime}\right)$ is continuous with respect to the semi-norm $\sqrt{D[f]}$. Now, by Lemma 1.3 in [18], we can find an element $g_{m}$ in $M_{0}^{n}\left(D_{m}\right)$ such that $\left\|g_{m}-\widetilde{g}_{m}\right\|<\varepsilon_{m}$ for an arbitrary given $\varepsilon_{m}$. Moreover, by the above, we can select $g_{m}$ so as to satisfy $\left\|g_{m} \circ T^{-1}-\widetilde{g}_{m} \circ T^{-1}\right\|<\varepsilon_{m}$. Then we see that

$$
\begin{aligned}
\sqrt{D\left[g_{m} \circ T^{-1}\right]} & >\sqrt{D\left[\widetilde{g}_{m} \circ T^{-1}\right]}-\varepsilon_{m}>\sqrt{2 m D\left[\tilde{g}_{m}\right]}-\varepsilon_{m} \\
& >\sqrt{2 m}\left(\sqrt{D\left[g_{m}\right]}-\varepsilon_{m}\right)-\varepsilon_{m} .
\end{aligned}
$$

If we choose $\varepsilon_{m}$ sufficiently small, then we have

$$
D\left[g_{m} \circ T^{-1}\right]>m D\left[g_{m}\right] \text { and } D\left[g_{m}\right]>1 .
$$

Next we choose a sequence $\left\{t_{m}\right\}$ such that $t_{m} D\left[g_{m}\right]=1 / m^{2}$. Then $0<t_{m}<1$. We put $g=\sum_{m} \sqrt{t_{m}} g_{m}$. Then $\sup _{U_{r}}|g|<1$ and $D[g]=\sum_{m} t_{m} D\left[g_{m}\right]=\sum_{m} 1 / m^{2}<\infty$. Hence $g$ is in $M\left(U_{r}\right)$. By defining $g=0$ outside $U_{r}$, we may consider $g$ to be in $M^{n}(S)$. It is well defined since $g$ vanishes in a neighborhood of the relative boundary of $U_{r}$ with respect to $S$.

By the definition of $T$, we have $g^{\circ}(T p)=g(p)$ on $U$. Then we get

$$
\begin{aligned}
D\left[g^{\circ}\right] & \geqq \iint_{U}\left|\operatorname{grad} g \circ T^{-1}\right|^{2} d x d y \\
& =\sum_{m} \iint_{D_{m}}\left|\operatorname{grad} \sqrt{t_{m}} g_{m} \circ T^{-1}\right|^{2} d x d y \\
& =\sum_{m} t_{m} D\left[g_{m} \circ T^{-1}\right] \geqq \sum_{m} m t_{m} D\left[g_{m}\right] \\
& =\sum_{m} 1 / m=\infty .
\end{aligned}
$$

which contradicts the condition $\left(\mathrm{M}^{n} .3\right)$, since $g^{\sigma}$ belongs to $M^{n}\left(S^{\prime}\right)$. Thus we have proved $K_{U_{r}}(T)<\infty$.

The sequence $\left\{K_{U_{r}}(T)\right\}_{r=1}^{\infty}$ is uniformly bounded. This can be proved by the similar method as above. 
Thus we see that the property $M^{n}$ satisfies (P.7). This can be proved by the similar method as in the last part of the proof of Lemma III.3. Hence we can apply Theorem $I .1$ to conclude that an arbitrary element in $I^{n}\left(R, R^{\prime}\right)$ is induced by a topological mapping $T$ of $R$ onto $R^{\prime}$, i.e., $f^{\sigma}(T p)=f(p)$ on $R$. Thus $T$ and $T^{-1}$ are $C^{n}$-mappings and the quasiconformality of $T$ can be concluded by the same method as above.

This completes the proof of Theorem III. 5 .

13. It is easy to see that if there exists a quasiconformal mapping of a Riemann surface $R$ onto another Riemann surface $R^{\prime}$, then there exists a $C^{\infty}$. quasiconformal mapping of $R$ onto $R^{\prime}{ }^{17)}$ From this and from Theorems III.1, III. 2 and III. 5 , we get the following

THEOREM III. 6. Two Riemann surfaces $R$ and $R^{\prime}$ are quasiconformally (resp. conformally) equivalent if and only if $M^{n}(R)$ and $M^{n}(R)$ are algebraically (resp. normed algebraically) isomorphic for some $n$ and hence for all $n=0,1,2, \ldots, \infty$.

\section{REFERENCES}

[1] L. Ahlfors: On quasiconformal mappings, J. d'Analyse Math., 3, 1-58, 207-208 (1953$1954)$.

[2] L. Bers: On rings of analytic functions, Bull. Amer. Math. Soc., 54, 311-315 (1948).

[3] L. Bers: On a theorem of Mori and the definition of quasiconformality, Trans. Amer. Math. Soc., 84, 78-84 (1957).

[4] N. Bourbaki: Topologie générale, Chap. X (1949).

[5] E. Čech: On bicompact spaces, Ann. of Math., 38, 823-844 (1937).

[6] I. Gelfand: Normierte Ringe, Rec. Math., 9, 3-24 (1941).

[7] M. Heins: Algebraic structure and conformal mapping. Trans. Amer. Math. Soc., 89, 267-276 (1958).

[8] E. Hewitt: Rings of real-valued continuous functions I, Trans. Amer. Math. Soc., 64, 45-99 (1948).

[9] T. Ishii: On homomorphisms of the ring of continuous functions onto the real numbers, Proc. Japan Acad., 33, 419-423 (1957).

[10] T. Ishiwata: On the ring of all bounded continuous functions, Sci. Rep. Tokyo Kyoiku Daigaku, 5, 279-280 (1957).

[11] T. Ishiwata: On locally Q-complete spaces, II, Proc. Japan Acad., 35, 263-267 (1959).

17) More strongly, we can prove the following: If $T$ is a quasiconformal mapping of a Riemann surface $R$ onto another Riemann surface $R^{\prime}$, then there exists a sequence of $C^{\infty}$-quasiconformal mappings $\left\{T_{n}\right\}_{n=1}^{\infty}$ such that $\lim _{B}\left[\sup _{B} \rho\left(T_{n}(p), T(p)\right)\right]=0$ and $\lim K\left(T_{n}\right)$ $=K(T)$, where $\rho$ is a metric on $R^{\prime}$ which induces the topology of $R^{\prime}$. In general, we cannot choose $\left\{T_{n}\right\}$ so as to satisfy $K\left(T_{n}\right) \leqq K(T)$ for all $n$. 
[12] S. Kakutani: On rings of analytic functions, Proc. Michigan Conference on functions of a complex variable (1953).

[13] L. Loomis: An introduction to abstract harmonic analysis (1953).

[14] A. Mori: On quasi-conformality and pseudo-analyticity, Trans. Amer. Math. Soc., 84, 56-77 (1957).

[15] S. Mori: A remark on a subdomain of a Riemann surface of the class $O_{H D}$, Proc. Japan Acad., 34, 251-254 (1958).

[16] S. Mori: On the ideal boundary of a simply connected open Riemann surface (in Japanese), Mem. Research Inst. Sci. and Eng., Ritsumeikan Univ., 2, 1-6 (1957).

[17] S. Mori and M. Ota: A remark on the ideal boundary of a Riemann surface, Proc. Japan Acad., 32, 409-411 (1956).

[18] M. Nakai: On a ring isomorphism induced by quasiconformal mappings, Nagoya Math. J., 14, 201-221 (1959).

[19] M. Nakai: A function algebra on Riemann surfaces, Nagoya Math. J., 15, 1-7 (1959).

[20] M. Nakai: Purely algebraic characterization of Quasiconformality, Proc. Japan Acad., 35, 440-443 (1959)

[21] A. Pfluger: Quasiconforme Abbildungen und logarithmische Kapazität, Ann. l'Inst. Fourier, 2, 69-80 (1951).

[22] A. Pfluger: Über die Äquivalenz der geometrischen und der analytischen Definition quasikonformer Abbildungen, Comment. Math. Helvet., 33, 23-33 (1959).

[23] E. Pursell: An algebraic characterization of fixed ideals in certain function rings, Pacific J. Math., 5, 963-969 (1955).

[24] H. Royden: The ideal boundary of an open Riemann surface, Annals of Mathematics Studies, 30, 107-109 (1953).

[25] H. Royden: A property of quasi-conformal mapping, Proc. Amer. Math. Soc., 5, 266269 (1954).

[26] H. Royden: Rings of analytic and meromorphic functions, Trans. Amer. Math. Soc., 83, 269-276 (1956).

[27] H. Royden: Open Riemann surfaces, Ann. Acad. Sci. Fenn., A.I. 249/5 (1958).

[28] H. Royden: Rings of meromorphic functions. Seminars on analytic functions, Inst. for Advanced study, 2, 273-285 (1958).

[29] W. Rudin: Some theorems on bounded analytic functions, Trans. Amer. Math. Soc., 78, 338-342 (1955).

[30] S. Saks: Theory of the integral, 2nd ed., Warsaw (1937).

[31] M. Shanks: Rings of functions on locally compact spaces, Bull. Amer. Math. Soc., 57, 295 (1951).

[32] T. Shirota: A generalization of a theorem of I. Kaplansky, Osaka Math. J., 4, 121132 (1952).

[33] J. Wermer: The maximum principle for bounded functions, Ann. of Math., 69, 598604 (1959).

[34] Z. Yujobo: On absolutely continuous functions of two or more variables in the Tonelli sense and quasiconformal mappings in the A. Mori sense, comment. Math. Univ. st. Pauli, 4, 67-92 (1955).

Mathematical Institute

Nagoya University 